\title{
Early rheumatoid arthritis: time to aim for remission?
}

\author{
Paul Emery, Michael Salmon
}

Rheumatoid arthritis (RA) is probably the commonest potentially treatable cause of disability in the western world. It is characterised by a symmetrical inflammatory synovitis which initially affects the metacarpal/metatarsal phalangeal (MCP/MTP) joints. Subsequently, this evolves to systemic disease with an increased acute phase response and extraarticular manifestations. In its mildest form, the synovitis may be non-destructive and self limiting, but the most severe form has a prognosis worse than that of malignancy. ${ }^{1}$ Early in disease, the symptoms and signs can be rapidly reversed by treatment such as corticosteroids. Once established, however, an almost inevitable progressive extension of the disease occurs, involving joint destruction and disability that lasts over the disease course of 20-25 years. The resultant morbidity and mortality produce a large social and economic cost. $^{2}$ Treatments used in the past showed short term benefit, though long term improvement in disease outcome has not been apparent; ${ }^{3}$ a truly effective treatment would have a significant impact on long term disability and costs. It is the contention of this article that the major factors that have contributed to this unsatisfactory situation have been a delay in presentation of patients to specialists, an inability to predict which patients were at risk of progressive joint disease, inadequate methods of monitoring early disease, and suboptimal use of existing treatments. The article reviews recent data relating particularly to prediction of outcome and the accurate monitoring of early disease and drug toxicity. Such information has enabled a radical approach to be formulated which, for the first time, has a realistic chance of substantially influencing the long term outcome of RA.

Rheumatology \&

Rehabilitation

University of Leeds

School of Medicine, 36 Clarendon Road, Leeds LS2 9NZ, United Kingdom P Emery

Department of Rheumatology, University of Birmingham, Edgbaston, Birmingham B15 2TT, United Kingdom M Salmon

Correspondence to: Professor P Emery.

Accepted for publication 26 July 1995

THE CRITICAL NATURE OF EARLY DISEASE AND WHY DELAY IN TREATMENT PRODUCES FUNCTIONAL DETERIORATION

The early phase of RA is a time of unique pathogenic importance. At onset, inflammation is at its peak: the rate of appearance of erosions, the number of inflamed joints, and the rate of bone loss in the axial skeleton (an objective marker of catabolism) are all maximal at this time, ${ }^{4}$ and it has been shown that patients with active disease over three years lose, on average, $20 \%$ of their bone mass from the hip. ${ }^{5}$ Patients who are often left untreated during this period of maximal inflammation are therefore likely to deteriorate, while suppressive treatment should be of greatest benefit at this time.

Treatment has a contrasting effect on inflammation and function. Inflammation responds to intervention with second line drugs with a steady improvement that is largely independent of the duration of disease. In contrast, the final functional status is determined by the duration of symptoms before initiation of therapy. ${ }^{4}$ Indeed, data from the review of our last 300 patients show that there is a level of disability in an individual above which reversibility is unlikely (unpublished). Thus, whilst inflammation is both treatable and reversible, the degree to which functional status can be improved is dependent on both its duration and extent. This has led to the concept, depicted in the figure, that early intervention is vital if long term disability is to be prevented.

\section{ADVANTAGE OF EARLY TREATMENT FOR} RHEUMATOID ARTHRITIS

There are a number of reasons to institute treatment early. First, an obvious but important one is that there is nothing to be gained by waiting. Recent evidence suggests that almost $90 \%$ of patients with RA will receive a second line drug in the first three years. The argument that the associated danger of toxicity should be avoided is not valid, as patients almost certainly will eventually require such a drug. ${ }^{6}$ The argument may now be more relevant for primary care physicians, encouraging them to refer patients early, than for rheumatologists, most of whom have accepted the argument that treatment should be initiated as soon as the diagnosis is made. Second, as mentioned above, on average, treatment produces only stabilisation of function, therefore if treatment is instigated early, before major disability occurs, there is a chance to stabilise patients functionally in a near normal state, rather than

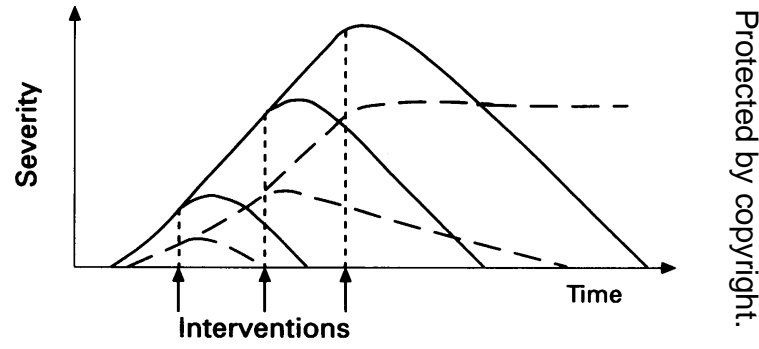

Effect of disease duration of rheumatoid arthritis on inflammation (-) and function (---). 
in a state of disability. Given that the mean duration of the disease is 25 years, this could have a major impact on the total disability. Furthermore, if patients are given drugs early rather than late in disease, when they are still relatively well, they are more likely to tolerate them. ${ }^{6}$ This may be because, when the disease mass is less, the acute phase response may not be increased; indeed more than $50 \%$ of early arthritis clinic referrals now present with normal values for $\mathrm{C}$ reactive protein, which becomes increased only as the disease (and synovial mass) progresses. This contrasts with established disease, in which the increased acute phase response is associated with alterations in hepatic cell function-providing a possible explanation for the poor tolerance of pharmacological intervention in established disease. ${ }^{6}$ Finally, extrapolating from the evidence from early treatment of the specific rheumatic diseases of oligo and monoarthritis, there is the possibility that the long term outcome may be altered. In a series of such patients treated early, those who received intraarticular steroid injections to the affected joints in the early months (and second line prophylaxis) did not have any further disease in their joints. $^{7}$ As the phenotype of $\mathrm{T}$ cells from synovial fluid is specifically that of recurrently activated memory cells, ${ }^{8}$ the effectiveness of intra-articular steroids may be attributable to the destruction of such memory $T$ cells. This may be the underlying basis of the concept of the therapeutic window-a longstanding idea that has been based on clinical observation.

\section{Problems with early presentation and treatment}

In a view of the above data, one might question why early treatment has not become routine. The major reasons are practical ones. First, whilst RA is common, it rarely presents early. The average time from first symptoms until the patient consults a rheumatologist is about nine to 12 months. The causes of this delay include the problem that the disease starts undramatically, affecting only the small joints in a way which is often not clearly arthritic. Patients frequently complain of stiffness of the hands and are often surprised to be told they have arthritis. Furthermore, if they are treated with a non-steroidal antiinflammatory drug their symptoms may temporarily disappear, only to re-emerge some time later. In addition, when only small joints are affected, patients may not have an increased acute phase response (purely because the amount of synovitis does not produce systemic levels of cytokines sufficient to activate the hepatocyte). Therefore it requires a very alert general practitioner to realise that the features he is seeing, namely reversibility with a non-steroidal drug (and dependence on it), plus the distribution of disease to the MCP and MTP joints, require an early referral. Many attempts to establish early arthritis clinics have failed because referrals have been dominated by chronic disorders other than inflammatory disease.
If patients are seen early, there is then the problem of how to distinguish between patients with RA and those with self limiting synovitis, including viral or reactive arthropathy: the former justify treatment with disease modifying drugs (DMARDs), whilst, generally, the latter do not. Clinical features alone do not allow an accurate prediction of outcome. ${ }^{9}$ The traditional approach was therefore to treat symptomatically and await the appearance of conventional markers indicating progressive disease. These markers, such as the involvement of large joints, an increased acute phase response, and the presence of radiological erosions, emerge relatively late in the evolution of the disease process, and by the time they are manifest the (poor) prognosis is already determined. This 'watch and wait' policy is unsatisfactory, and may even contribute to the poor prognosis by delaying treatment. A means of accurate prediction of outcome is necessary if patients are to be treated appropriately at an eary stage.

Even when patients are seen and treated at an early stage of disease, there is the further problem that there is no convenient method of monitoring change in disease activity and outcome. Conventional measures of disease activity-for example acute phase response or outcome measures such as functional status and radiological erosions-are not appropriate if patients are seen earlier in the disease process, as these markers may be normal. In a survey of our most recently presenting patients, more than $50 \%$ had a normal acute phase response, and the majority also showed no radiological evidence of joint erosion. If early intervention is to be used, a means of monitoring its effect is required.

Finally, there is considerable debate about the efficacy of so called ('disease modifying') DMARDs. Recently, this debate has shifted from whether they are effective at all, to whether they have any long term benefit. Again, this may simply reflect delay in their use.

\section{Solutions to the problems preventing a better clinical outcome}

EARLY REFERRAL BEFORE FUNCTIONAL DETERIORATION

In an attempt to ensure that patients are referred at a stage in their disease before functional deterioration has ocurred, specialist early arthritis clinics have been established. These have been combined with an education programme intended to increase awareness among general primary care physicians of the poor prognosis of untreated disease, indicating those features that are compatible with inflammatory arthritis, and reinforcing the importance of not waiting for features which are markers of late disease (for example increased erythrocyte sedimentation rate, erosions). Particular emphasis was put on the fact that a response to a non-steroidal drug indicated reversibility of disease requiring urgent referral, rather than the reverse. The concept conveyed to general practitioners was one of an early 
phase of intensive disease management which would be followed by shared care. As a consequence of such a programme, the delay between appearance of the first symptoms and the patient being seen by a specialist has decreased from 14 months in 1988 to three months in 1993.

PREDICTION OF OUTCOME AT PRESENTATION The ability to identify patients at risk of joint destruction can be improved by the use of genetic markers such as major histocompatibility class II typing. The potential value of this was initially described in a research clinic $^{10}$ and, subsequently, the hypothesis that possession of the so called 'conserved epitope' would predict severity of disease was validated in a large group of consecutive patients attending a routine clinic. ${ }^{11}$ The important findings were that possession of a conserved amino acid sequence in the third hypervariable region of the DR $\beta 1$ allele, though not necessarily predisposing to disease, was very important in determining the disease severity and, when combined with the presence of rheumatoid factor, permitted clinically useful predictions to be made. Using just these two markers it was possible to predict with a high sensitivity (relative risk 13.5) or high specificity (relative risk $8 \cdot 8$ ) which patients would develop radiographically detectable joint erosion during a period of one year. ${ }^{5}$ This is of clinical use, in that sensitive markers can be used to identify patients suitable for treatment with a safe drug, or specific markers can be used to select patients for a more rigorous disease suppressive regimen. This indicator system also has the great advantage of being independent of the stage of disease, and therefore applicable to early disease (rheumatoid factor is positive at presentation in the majority of patients in whom it will later be recognised as significantly positive). A subgroup of patients were found to have two disease associated HLA allelesone at each chromosomal locus; the prognosis of these patients was particularly poor. Rheumatoid factor has routinely been used to stratify patients for treatment but, until recent data revealed its value, the disease epitope had not been used prospectively for such a purpose.

MONITORING OF EARLY DISEASE

Monitoring established disease is straightforward, because of the large number of secondary abnormalities, such as acute phase response and abnormal radiology. Early disease is different, in that more than $50 \%$ of patients do not have an increased acute phase response, and most have no radiological destruction at presentation. Conventional techniques are therefore ineffective for monitoring early disease. However, it is well recognised that periarticular osteoporosis of the hands represents the first objective sign of disease, and recent work has shown that measurements of the hand, made on dual energy $x$ ray absorption (DXA) scans are both reproducible and highly sensitive, with changes detectable over periods as short as three months. ${ }^{12}$ As the major factors influencing periarticular osteoporosis are disease activity and function of the hand, and these are the factors that treatment aims to influence (by suppressing disease and maintaining function), this measure has the potential to be an ideal indicator of outcome. ${ }^{12}$ This technique should allow assessment of patients at presentation, including those with a grumbling onset of disease for whom no other outcome measure is currently available. In addition, axial bone densitometry has shown its potential to distinguish between the divergent systemic effects of corticosteroids (total dose given by all routes) on outcome: while steroid use improves the major determinants of bone loss (inflammatory disease activity and loss of function) the negative catabolic effects of corticosteroids contribute to bone loss. ${ }^{5}$ Thus an improvement in bone mineral density in patients receiving corticosteroids provides reassurance that the positive effects of corticosteroids are outweighing the negative.

\section{EFFECTIVE TREATMENTS}

Realisation of the importance of the early phase of disease has led to the alternative strategy of treating all patients aggressively from the onset. This, however, may exchange the adverse effect of delayed treatment for increased adverse reactions to treatment in patients who have been given the drugs unnecessarily. To be most cost effective, it is necessary to treat patients in a targeted fashion, restricting aggressive regimens to those patients who are likely to deteriorate. Corticosteroids remain the most effective treatment in RA and can be used selectively intra-articularly, possibly inducing programmed cell death. ${ }^{13}$ If ablation of $T$ memory cells is combined with treatment that prevents $T$ cell accumulation or activation in the joints, it should be possible to suppress disease in a manner analogous to that of organ transplantation. There is increasing evidence of the efficacy of combined treatments in established disease, ${ }^{14}$ and it is likely that a variety of approaches will produce positive results. Specific (but expensive) treatment regimens that are targeted at particular points in the inflammatory process and which could be used as part of such an approach are already available. ${ }^{15} 16$ Recent data from randomised studies have shown the ability of cyclosporin to act additively with methotrexate without increased toxicity ${ }^{14}$ and, when given in early disease, to prevent the appearance of new erosions. ${ }^{17}$ The imminent licensing of tenidap will add further possibilities, as this has also been shown to retard erosions. ${ }^{18}$

All the elements necessary for a radical approach to treatment are thus now in place:

(1) There is unequivocal evidence that conventionally treated patients have a poor prognosis. $^{3}$

(2) Specialists are seeing patients before functional deterioration has occurred (unpublished observation, Early Arthritis Clinic). 
(3) At last, hand DXA provides a satisfactory outcome measure for monitoring early disease in patients who do not have radiological erosions or functional deterioration. ${ }^{12}$

(4) Axial DXA measurement provides a method of assessing the toxicity of corticosteroids that will be a necessary part of any disease suppressant regimen. ${ }^{5}$

(5) Clinically useful prediction has been demonstrated. ${ }^{11}$

(6) Suitable disease suppressive procedures are now available. ${ }^{14-18}$

Centres capable of genetically typing patients and having the tools to monitor them effectively can use this approach to provide a staging classification of early disease. ${ }^{4}$ These centres should be comparing current best treatment with procedures aiming to produce remission for a substantial proportion of patients. A logical approach using currently licensed drugs would be to induce remission with intra-articular corticosteroids and provide maintenance treatment with a combination of cyclosporin and methotrexate. ${ }^{14}$ This clearly needs to be under carefully controlled conditions, and a study of such a regimen is now in progress. The results should give a rapid, clear indication of the feasibility of this approach and a cost-effectiveness or a cost-utility analysis (or both) will be among the most important measures of outcome. The cost of tissue typing (currently around $£ 40$ in the UK) is likely to be an irrelevance in a disease of prolonged duration with an estimated annual cost of more than 200 times that (US data). The extra costs of monitoring in any subsequent study would be reduced, as the current DXA imaging is required for efficacy and toxicity analysis in this primary study, but will not be required routinely in subsequent protocols.

It is notable that all newly diagnosed cancer patients are recorded on a register in a model system that should be copied by physicians managing patients with RA. The latter patients suffer a disease with a duration of 25 years and a failure to diagnose correctly and treat appropriately at a time when function is normal can lead to enormous costs for both patient and society. It is crucial that patients with this expensive disease are not managed randomly, but that serious attempts are made to reduce these social costs. For the first time, the obstacles which have prevented such an approach have been removed.

1 Pincus T, Callahan L F, Sale W G, Brooks A L, Payne L E, Vaughn W K. Severe functional declines, work disability, and increased mortality in seventy-five rheumatoid arthritis patients studied over nine years. Arthritis Rheum 1984; 27: 864-72.

2 Rasker J S, Cosh J A. National history of rheumatoid arthritis-15 year follow-up study. The prognostic significance of features noted in the first year. Clin Rheumatol 1984; 111-20.

3 Scott D L, Symmons D P M, Coulton B L, Popert A J. Long-term outcome of treating rheumatoid arthritis: results after 20 years. Lancet 1987 ; 1: 1108-11.

4 Emery P. The optimal management of early rheumatoid arthritis: the key to preventing disability [The Roche Prize Lecture]. Br f Rheumatol 1994; 33/8: 765-8.

5 Gough A K, Lilley J, Eyre S, Holder R L, Emery P. Generalised bone loss in patients with early rheumatoid arthritis occurs early and relates to disease activity. Lancet 1994; 344: 23-7.

6 Young A, Cox N, Davis P, et al. Treatment patterns over 2 years in 577 patients with rheumatoid arthritis of recent
oung $A$, Cox $N$, Davis $P$ al 2 years in 577 patients with rheumatoid arthritis
onset. Arthritis Rheum 1994; 37 (suppl 9): S258.

7 Devlin J, Gough A, Huissoon A, Perkins P, Jubb R, Emery P. Knee disease in 766 early arthritis patients: relevance to outcome and pathogenesis [abstract]. Arthritis Rheum 1993; 36 (suppl 9): 262.

8 Matthews N, Emery P, Pilling D, Akbar A, Salmon M. Subpopulations of primed T helper cells in RA. Arthritis Rheum 1993; 36: 603-7.

9 Tunn E J, Bacon P A. Differentiating persistent from selflimiting symmetrical synovitis in an early arthritis clinic. Br ₹ R heumatol 1993; 32: 97-103.

10 Emery P, Salmon M, Bradley $\mathrm{H}$, et al. Genetically determined factors as predictors of radiological change in patients

11 Gough A K, Faint J M, Salmon M, et al. Genetic typing of patients with inflammatory arthritis at presentation is predictive of outcome. Arthritis Rheum 1994; 37: 1166-70.

12 Devlin J, Lilley J, Huissoon A, et al. Hand DXA-the ideal outcome measure? Correlation with hand function and disease activity. Arthritis Rheum 1994; 37 (suppl 9): S251.

13 Liuz G, Smith S W, McLaughlin K A, Schwartz L M, Osborne B A. Apoptotic signals delivered through the $T$ cell receptor of a $\mathrm{T}$ cell hybrid require the immediate/early gene Nur77N. Nature 1994; 367: 281-4.

14 Tugwell $\mathrm{P}$, Pincus T, Yocum D, et al. A multi-centre doubleblind, randomised trial of low-dose cyclosporin and placebo therapy in combination with methotrexate in placebo therapy in combination with methotrexate in patients with severe

15 Kavanaugh A F, David L S, Nichols L A, et al. Treatment of refractory rheumatoid arthritis with a mononuclear antibody to intercellular adhesion molecule 1. Arthritis Rheum 1994; 37: 992-9.

16 Elliott M J, Maini R N, Feldman M, et al. Randomised double-blind comparison of chimeric monoclonal antibody to tumour necrosis factor a (cA2) versus placebo in rheumatoid arthritis. Lancet 1994; 344: 1105-10.

17 Portioli I, Pipitone V, Magaro M, et al. Low dose cyclosporin versus conventional second line drugs in patients with early active RA [EULAR abstract]. Rheumatology in Europe 1995; 24 (suppl 3): 231 (abs D60).

18 Genant H K, Kirby D S, Pickering E A, et al. Tenidap slows $\mathrm{X}$-ray progression in RA [EULAR abstract]. Rheumatology in Europe 1995; 24 (suppl 3): 224 (abs D30). 